\title{
Discrete chaos in a novel two-dimensional fractional chaotic map
}

Jie $\operatorname{Ran}^{1 *}(1)$

\author{
"Correspondence: \\ ranjie93@163.com \\ 'School of Mathematics, Zunyi \\ Normal College, Zunyi, China
}

\begin{abstract}
In this paper, a two-dimensional discrete fractional reduced Lorenz map is achieved by utilizing discrete fractional calculus. By adopting the bifurcation diagrams, chaos diagram, and phase portraits, the chaotic dynamics of the two-dimensional discrete fractional reduced Lorenz map are analyzed. Complexity of this fractional map versus parameters is discussed by employing the $C_{0}$ algorithm. It is found that this fractional map has rich dynamical behaviors. In addition, it also shows that the $C_{0}$ algorithm provides a parameter choice method for practice applications of discrete fractional maps. Finally, some numerical simulations are given to demonstrate the effectiveness of the proposed results.
\end{abstract}

Keywords: Discrete fractional calculus; Caputo-like delta difference; Chaos; $C_{0}$ algorithm

\section{Introduction}

In the past five decades, fractional-order chaotic systems have received increasing attention since fractional derivatives provide a memory effect. Many important and interesting research work on the chaotic dynamics of fractional-order chaotic systems can be found in $[1-3]$.

However, most of these results are concentrated on fractional-order differential equations, and there is little discussion for discrete fractional chaotic systems. For the basic definitions about discrete fractional calculus, readers can refer to references [4-9]. Recently, Baleanu and Wu et al. have achieved a series of important results. For example, discrete chaos in fractional sine and standard maps is researched by Wu et al. [10]. In [11] the chaos synchronization of the discrete fractional logistic map is discussed. In addition, a lot of important research about the dynamics of discrete fractional equations can be found in [12-24] and the references therein.

Although many results have been presented, the chaotic dynamics, especially, the identification of chaos and bifurcation behaviors, in the discrete fractional chaotic systems are still kept open and deserve further investigation.

Recently, many research results have been proposed about the complexity measure of chaotic sequences $[25,26]$ and the references cited therein. However, the complexity measure of chaotic sequences in the discrete fractional difference systems have been rarely reported. So it is an interesting topic for us to explore the complexity in the discrete fractional chaotic systems. Based on this, in this paper, I extended the previous research works

(c) The Author(s) 2018. This article is distributed under the terms of the Creative Commons Attribution 4.0 International License (http://creativecommons.org/licenses/by/4.0/), which permits unrestricted use, distribution, and reproduction in any medium, provided you give appropriate credit to the original author(s) and the source, provide a link to the Creative Commons license, and indicate if changes were made. 
for discrete fractional chaotic systems. The $C_{0}$ algorithm $[25,26]$ is introduced into the discussion of a discrete fractional chaotic map. In this article, a discrete reduced Lorenz map is given as a case (for the discrete reduced Lorenz map, readers can refer to [27-29]).

This paper is organized as follows. In Sect. 2, we introduce some of basic definitions and the preliminaries of the discrete fractional calculus, and the fractional reduced Lorenz map is described. In Sect. 3, the chaotic behaviors are reported and the bifurcation diagrams are given for various difference orders. In Sect. 4 , the $C_{0}$ complexity is calculated in detail. The conclusions are presented in the last section.

\section{Problem description and preliminaries}

In this section, in order to understand the analysis of fractional-order discrete chaotic system, I give a brief introduction for some basic definitions and properties of fractional integrals and derivatives. And a novel two-dimensional discrete fractional model is obtained by employing the left Caputo-like delta difference.

\subsection{Discrete fractional calculus: basic notions}

I start with some necessary definitions from discrete fractional calculus theory and review the preliminary results so that paper is self-contained [19]. The general $n$th order difference can be written as

$$
\Delta^{n} x(t)=\Delta^{n-1} x(t+1)-\Delta^{n-1} x(t)=\sum_{k=0}^{n} C_{n}^{k}(-1)^{k} x(t+n-k) .
$$

Extending this concept for fractional-order difference, the fractional sum of order $\alpha$ is defined as follows.

Definition 1 ([6]) $x: N_{\theta} \rightarrow R$ and $\alpha>0$, the fractional sum of order $\alpha$ is defined by

$$
\Delta_{\theta}^{-\alpha} x(t):=\frac{1}{\Gamma(\alpha)} \sum_{s=\theta}^{t-\alpha}\left(t-\sigma(s)^{\alpha-1}\right) x(s), \quad t \in N_{\theta+\alpha},
$$

where $\theta$ is the starting point, $\sigma(s)=s+1$, and $t^{(\alpha)}$ is the falling function defined as $t^{(\alpha)}=$ $\frac{\Gamma(t+1)}{\Gamma(t+1-\alpha)}$.

Definition $2([7,8])$ For $\alpha>0$ and $x(t)$ defined on $N_{\theta}$, the $\alpha$ th Caputo-like delta difference is defined as

$$
{ }^{C} \Delta_{\theta}^{\alpha} x(t):=\frac{1}{\Gamma(m-\alpha)} \sum_{s=\theta}^{t-(m-\alpha)}(t-\sigma(s))^{(m-\alpha-1)} \Delta^{m} x(s), \quad t \in N_{\theta+m+\alpha},
$$

where $m=[\alpha]+1$, the $[\cdot]$ denotes the ceiling of number.

Remark 1 If $m=1$, the $\alpha$ th Caputo-like delta difference is defined by

$$
{ }^{C} \Delta_{\theta}^{\alpha} x(t):=\frac{1}{\Gamma(1-\alpha)} \sum_{s=\theta}^{t-(1-\alpha)}(t-\sigma(s))^{(-\alpha)} \Delta x(s), \quad t \in N_{\theta+1-\alpha} .
$$


Theorem 1 ([9]) For the delta fractional difference equation

$$
\begin{aligned}
& { }^{C} \Delta_{\theta}^{\alpha} x(t)=f(t+\alpha-1, x(t+\alpha-1)), \\
& \Delta^{(k)} x(\theta)=x_{k}, \quad m=[\alpha]+1, k=0,1,2 m, \ldots, m-1,
\end{aligned}
$$

the equivalent discrete integral equation can be obtained as

$$
x(t)=x_{0}(t)+\frac{1}{\Gamma(\alpha)} \sum_{s=\theta+m-\alpha}^{t-\alpha}\left(t-\sigma(s)^{\alpha-1}\right) \times f(s+\alpha-1, x(s+\alpha-1)), \quad t \in N_{\theta+m},
$$

where the initial iteration reads

$$
x_{0}(t)=\sum_{k=0}^{m-1} \frac{(t-\theta)^{(k)}}{k !} \Delta^{k} x(\theta) .
$$

Remark 2 If the initial point $\theta=0$, and limiting ourselves to $0<\alpha<1$, Eq. (6) changes to

$$
x(t)=x_{0}(t)+\frac{1}{\Gamma(\alpha)} \sum_{s=1-\alpha}^{t-\alpha}(t-\sigma(s))^{(\alpha-1)} \times f(s+\alpha-1, x(s+\alpha-1)),
$$

with $s+\alpha=j$, and using the expansion $(t-\sigma(s))^{(\alpha-1)}=\frac{\Gamma(t-s)}{\Gamma(t-s-\alpha+1)}$, as a result, the numerical formula can be presented explicitly

$$
x(t)=x_{0}(t)+\frac{1}{\Gamma(\alpha)} \sum_{j=1}^{t} \frac{\Gamma(t-j+\alpha)}{\Gamma(t-j+1)} \times f(j-1, x(j-1)) .
$$

\subsection{Discrete fractional reduced Lorenz map and its solution}

This section illustrates the application of the Caputo-like delta difference to a discrete reduced Lorenz map. The discrete reduced Lorenz map is a discretization of a pair of coupled differential equations which were used by Lorenz et al. [27-29]. The discrete reduced Lorenz map is defined as follows:

$$
\left\{\begin{array}{l}
x(t+1)=(1+a h) x(t)-h x(t) y(t) \\
y(t+1)=(1-h) y(t)+h x(t)^{2}
\end{array}\right.
$$

The fractional-order version of a discrete reduced Lorenz map has not been studied much. By adopting the Caputo-like delta difference, the discrete fractional reduced Lorenz map is obtained as follows:

$$
\left\{\begin{array}{l}
{ }^{C} \Delta_{\theta}^{\alpha} x(t)=(1+a h) x(t+\alpha-1)-h x(t+\alpha-1) y(t+\alpha-1)-x(t+\alpha-1), \\
{ }^{C} \Delta_{\theta}^{\alpha} y(t)=(1-h) y(t+\alpha-1)+h x(t+\alpha-1)^{2}-y(t+\alpha-1) .
\end{array}\right.
$$

According to Theorem 1, the numerical solution is given as

$$
\left\{\begin{array}{l}
x(t)=x(\theta)+\frac{1}{\Gamma(\alpha)} \sum_{j=\theta+1}^{t} \frac{\Gamma(t-j+\alpha)}{\Gamma(t-j+1)} \times((1+a h) x(j-1)-h x(j-1) y(j-1)-x(j-1)), \\
y(t)=y(\theta)+\frac{1}{\Gamma(\alpha)} \sum_{j=\theta+1}^{t} \frac{\Gamma(t-j+\alpha)}{\Gamma(t-j+1)} \times\left((1-h) y(j-1)+h x(j-1)^{2}-y(j-1)\right),
\end{array}\right.
$$


where $x(\theta)$ and $y(\theta)$ are the initial conditions which can be defined, respectively. Especially, if $\theta=0$, (12) can be written as

$$
\left\{\begin{array}{l}
x(t)=x(0)+\frac{1}{\Gamma(\alpha)} \sum_{j=\theta+1}^{t} \frac{\Gamma(t-j+\alpha)}{\Gamma(t-j+1)} \times((1+a h) x(j-1)-h x(j-1) y(j-1)-x(j-1)), \\
y(t)=y(0)+\frac{1}{\Gamma(\alpha)} \sum_{j=\theta+1}^{t} \frac{\Gamma(t-j+\alpha)}{\Gamma(t-j+1)} \times\left((1-h) y(j-1)+h x(j-1)^{2}-y(j-1)\right) .
\end{array}\right.
$$

\section{Chaos of a discrete fractional reduced Lorenz map}

In this section, by means of phase portraits and bifurcation diagrams, the dynamics of system (11) are studied. Some numerical simulation results are obtained and some stronger chaotic attractors in system (11) introduced above are shown.

Variations of the fractional order $\alpha$ have been considered by keeping $h=0.95$ and $a=1$. The bifurcation diagram of system (11) is obtained as shown in Fig. 1. Letting $\alpha=1$, the phase portrait of system (11) in Fig. 2(a) is obtained, and the corresponding solutions are given in Fig. 2(b). By increasing the value of $\alpha$, when $\alpha=0.3,0.5,0.75,0.81,0.95$, respectively. The phase portraits of system (11) are obtained as shown in Figs. 2(c, e, g, i, k). The corresponding solutions are given in Figs. 2(d, f, h, j, l), respectively. From Figs. 1 and 2, it is clear that stable, periodic, quasi-periodic solutions, and ensuing transition to chaos can be observed. Figure 3 shows the bifurcation diagram for when fractional order is fixed as $\alpha=0.95$ and parameter $a$ is varied. Similarly, when parameter $b$ is varied, the bifurcation diagram is shown in Fig. 4.

Based on the above analyses, we can observe that the chaotic zones are clearly dependent on the changing difference order $\alpha$. These observations convey that variety of chaotic attractors can be obtained by varying fractional order and system parameters [15]. As it can be seen from Fig. 3, the periodic behaviors occur when $a<0.81$. For $a \geq 0.81$, system (11) exhibits chaos. From Fig. 4 , the periodic behaviors occur when $h<0.77$. For $h \geq 0.77$, system (11) exhibits chaos. By taking $a=0.81$, the phase portrait is depicted in Fig. 5(a). Let $h=0.77$, the phase portrait is depicted in Fig. 5(b).

\section{Complexity analysis}

In this section, the $C_{0}$ complexity of system (11) is discussed by employing the $C_{0}$ algorithm $[25,26]$, which is briefly described as follows.

Figure 1 Bifurcation diagram. The bifurcation diagram with variation in parameter $\alpha$ for system (11) for $a=1, h=0.95$

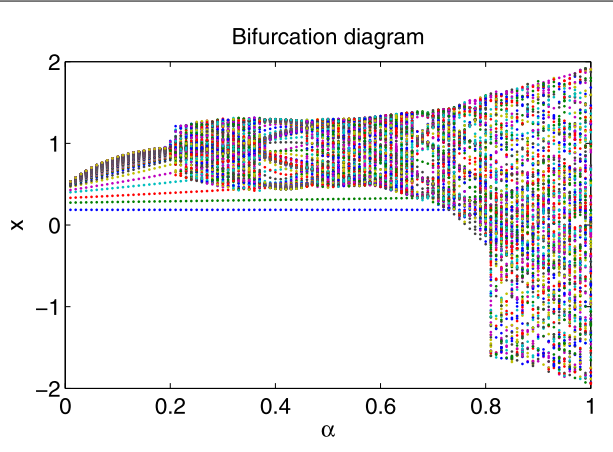




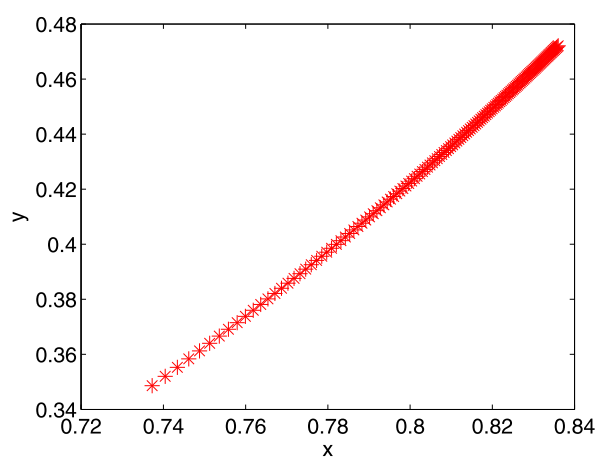

(a)

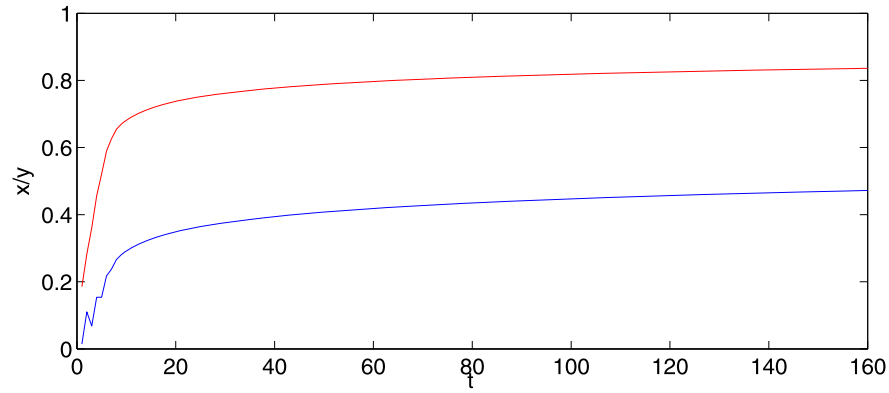

(b)

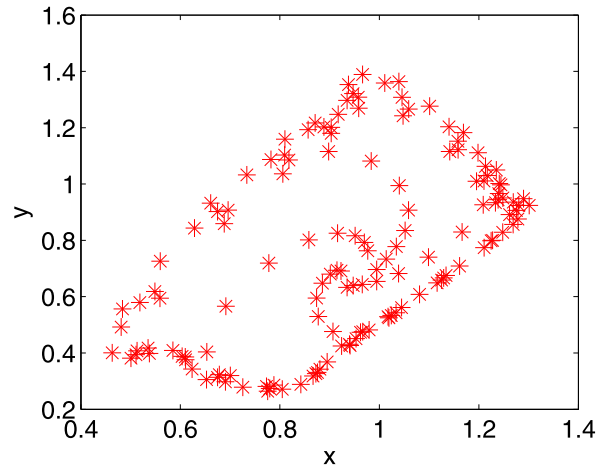

(c)

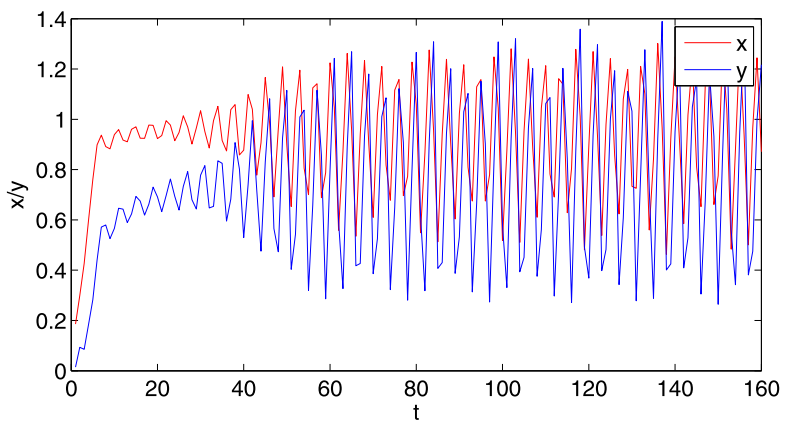

(d)

Figure 2 Phase portraits and their corresponding chaotic solutions. Phase portraits and solutions of system (11) with $a=1, h=0.95$, and different values of $\alpha$. (a, b) $\alpha=1$, (c, d) $\alpha=0.3$, (e, f) $\alpha=0.5,(\mathbf{g}, \mathbf{h}) \alpha=0.75$, $(\mathbf{i}, \mathbf{j}) \alpha=0.81,(\mathbf{k}, \mathbf{l}) \alpha=0.95$ 


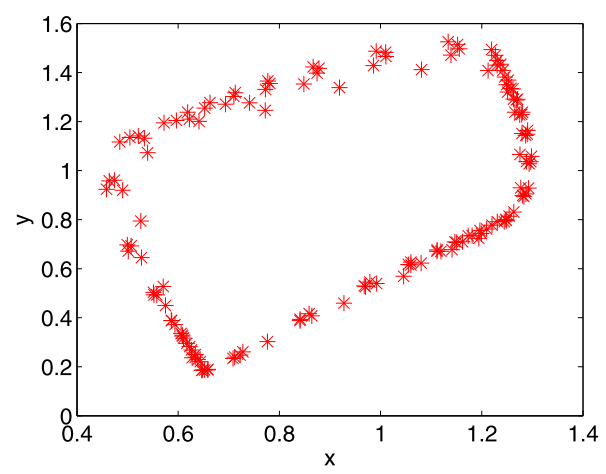

(e)

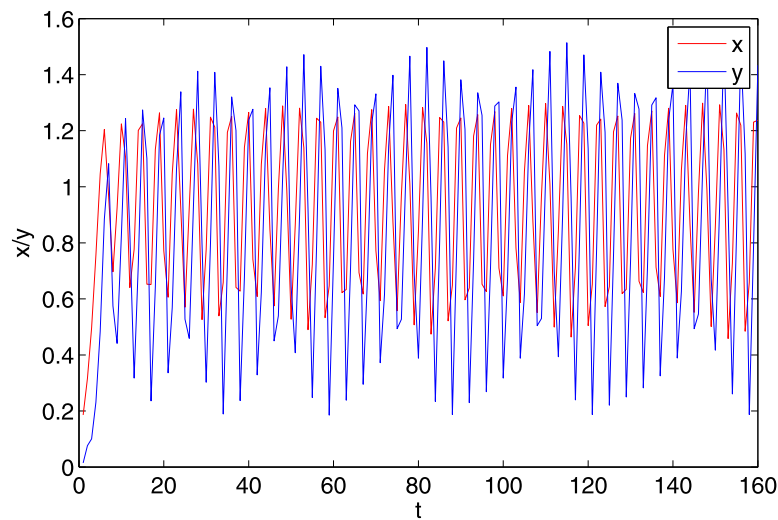

(f)

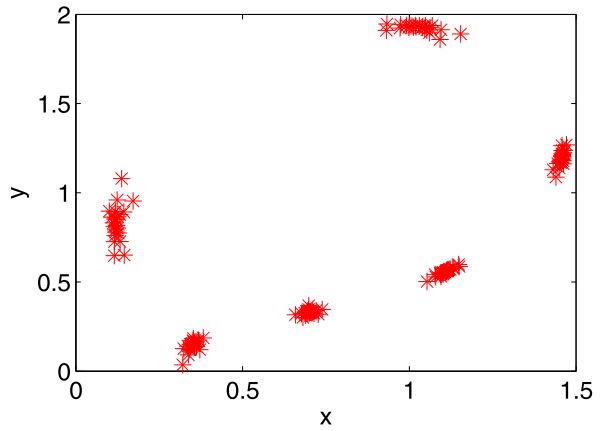

(g)

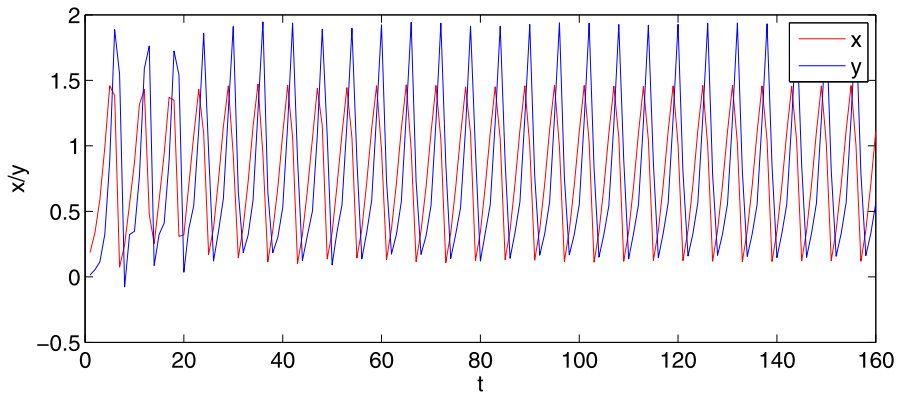

(h)

Figure 2 Continued 


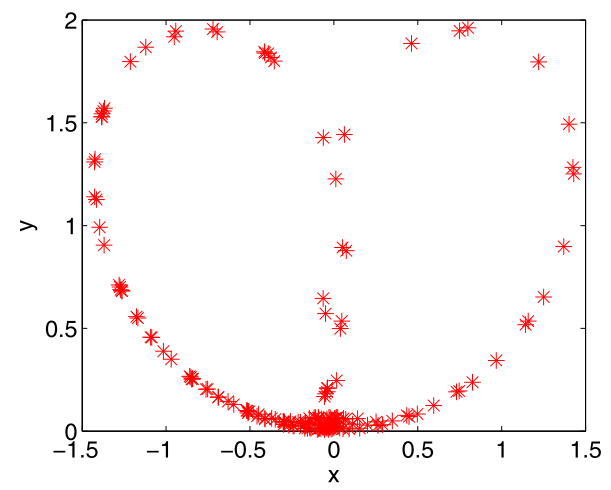

(i)

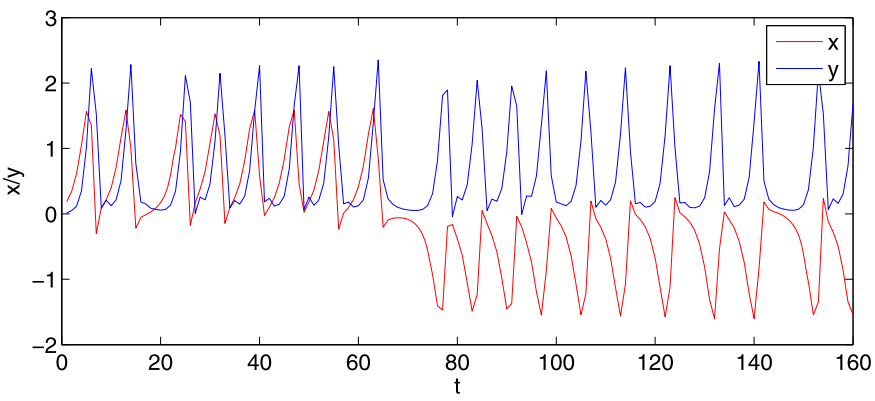

(j)

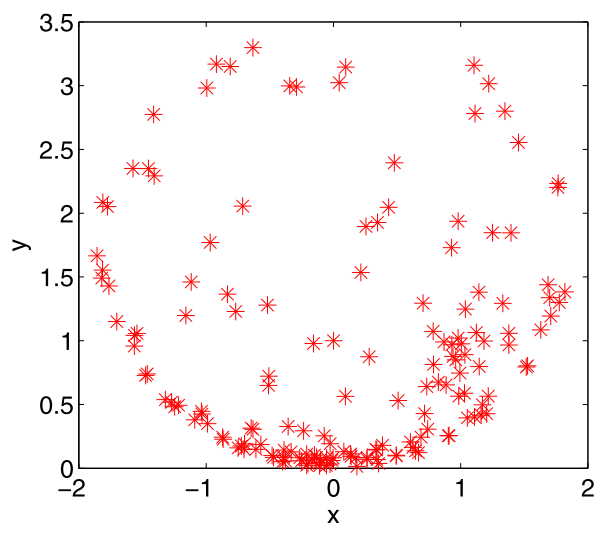

(k)

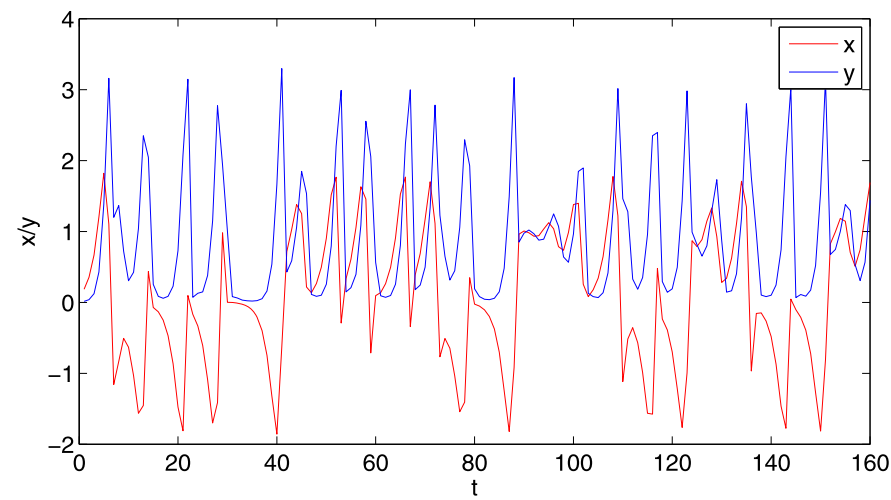

(l)

Figure 2 Continued 
Figure 3 Bifurcation diagram. The bifurcation diagram with variation in parameter $\alpha$ for system (11) for $\alpha=0.95, h=0.95$

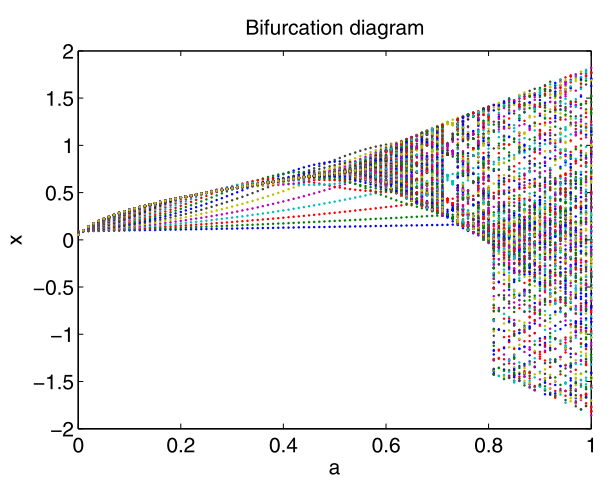

Figure 4 Bifurcation diagram. The bifurcation diagram with variation in parameter $h$ for system (11) for $\alpha=0.95, a=1$

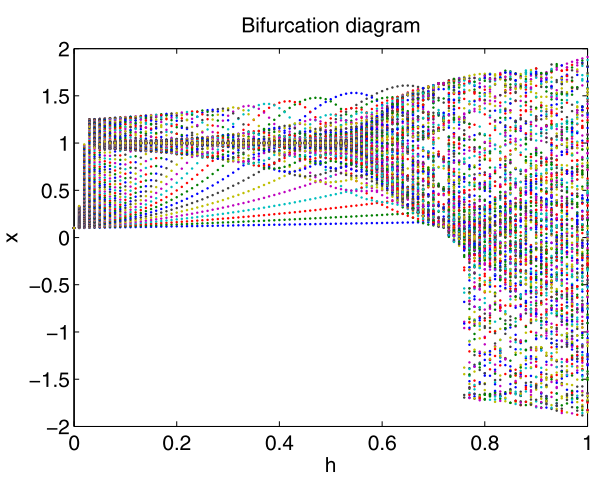

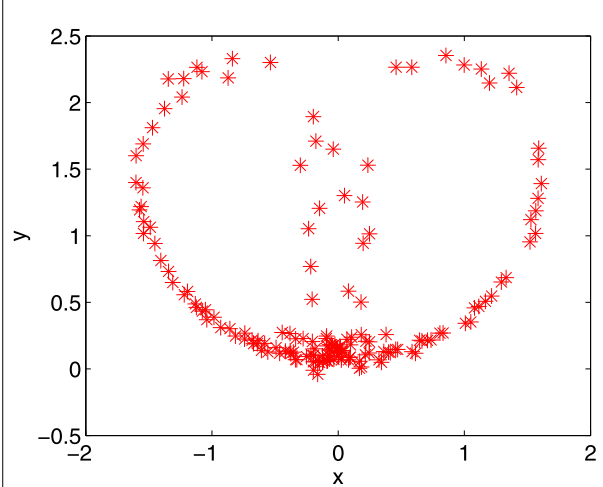

(a)

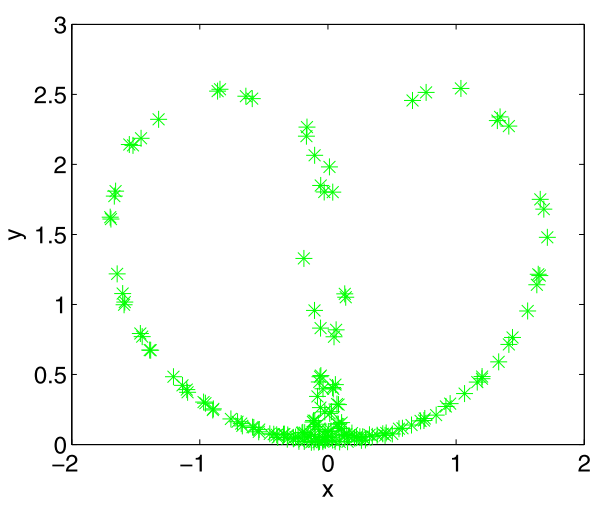

(b)

Figure 5 Phase portraits. The phase portraits of system (11) for $\alpha=0.95$, (a) $a=0.81, h=0.95$, (b) $a=1$, $h=0.77$

Consider a set of discrete data $\{\phi(n), n=0,1,2, \ldots, N-1\}$, and its corresponding discrete Fourier transform is

$$
\Phi(k)=\sum_{n=0}^{N-1} \phi(n) e^{\frac{-j 2 n k \pi}{N}}
$$




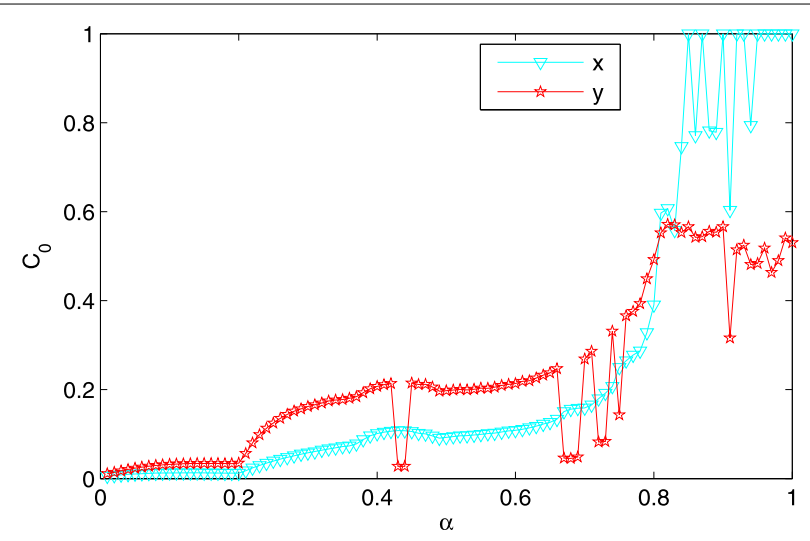

(a)

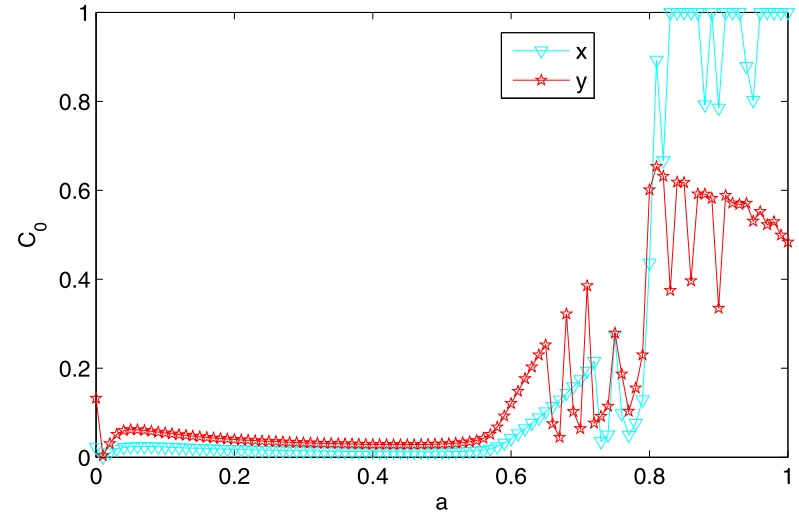

(b)

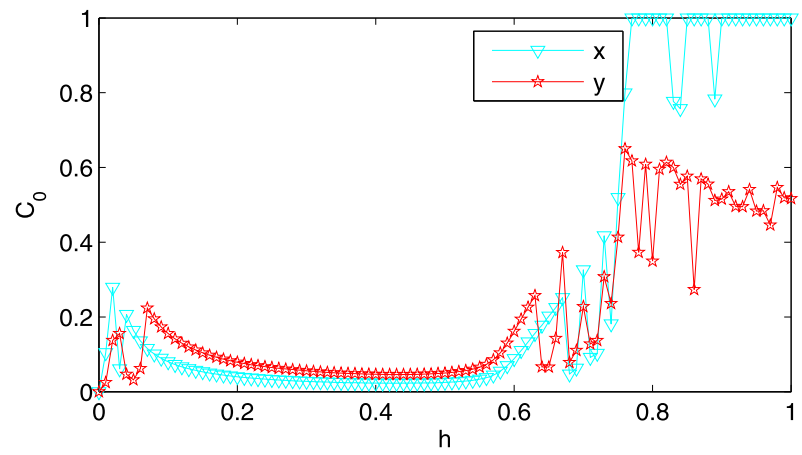

(c)

Figure $6 C_{0}$ complexity. (a) The $C_{0}$ complexity of system (11) with $a=1, h=0.95$, and $\alpha \in(0,1]$. (b) The $C_{0}$ complexity of system (11) with $\alpha=0.95, h=0.95$, and $a \in[0,1]$. (c) The $C_{0}$ complexity of system (11) with $\alpha=0.95, a=1$, and $h \in(0,1]$

where $k=0,1,2, \ldots, N-1$ and $j$ is the imaginary unit. The mean square value of $\{\Phi(k), k=$ $0,1,2, \ldots, N-1\}$ is defined as

$$
G_{N}=\frac{1}{N} \sum_{k=0}^{N-1} \phi(n) e^{\frac{-j 2 n k \pi}{N}}
$$




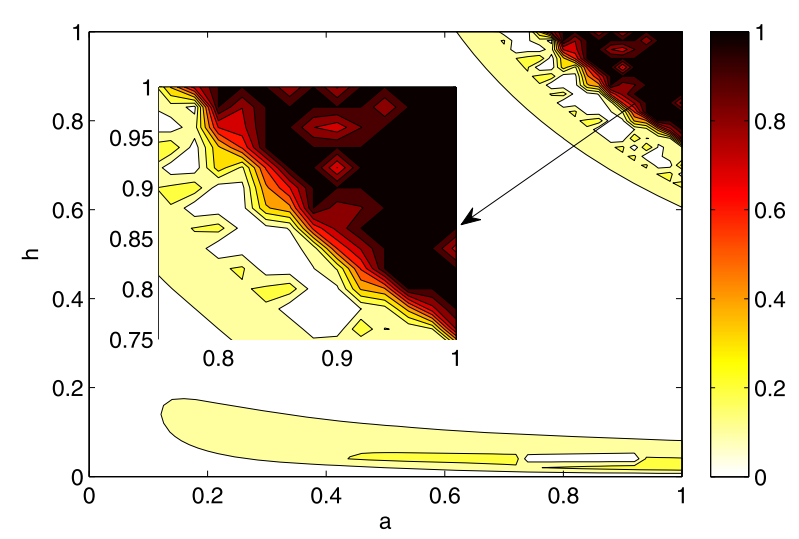

Figure 7 Chaos diagram. The chaos diagram of $C_{0}$ complexity of system (11) with $\alpha=0.95$ in the $(a, h)$ parameter plane

Let

$$
\bar{\Phi}(k)= \begin{cases}\Phi(k), & \text { if }|\Phi(k)|^{2}>r G_{N} \\ 0, & \text { if }|\Phi(k)|^{2} \leq r G_{N}\end{cases}
$$

where $r$ is a control parameter, and I choose $r=15$ in this paper. The inverse Fourier transformation of $\bar{\phi}(k)$ is

$$
\bar{\phi}(k)=\frac{1}{N} \sum_{k=0}^{N-1} \bar{\Phi}(k) e^{\frac{j 2 n k \pi}{N}}=\frac{1}{N} \sum_{k=0}^{N-1} \bar{\Phi}(k) W_{N}^{-n k},
$$

where $n=0,1,2, \ldots, N-1$. Then the $C_{0}$ complexity $[25,26]$ is defined as

$$
C_{0}(r, N)=\frac{\sum_{n=0}^{N-1}|\phi(n)-\bar{\phi}(n)|^{2}}{\sum_{n=0}^{N-1}|\phi(n)|^{2}} .
$$

In this study, I choose parameter planes $(a, b),(a, \alpha)$, and $(b, \alpha)$ to study their $C_{0}$ complexity. By taking $a=1$ and $h=0.95$, the $C_{0}$ complexity of system (11) is calculated and illustrated in Fig. 6(a). It can be seen that the $C_{0}$ complexity increases with increasing of fractional order $\alpha$, the $C_{0}$ complexity is 1 , when $\alpha \in[0.85,1]$, roughly. Fixing $\alpha=0.95$ and $h=0.95$, the $C_{0}$ complexity is calculated by varying the parameter $a$ in the range $[0,1]$ and illustrated in Fig. 6(b). Keeping $\alpha=0.95, a=1$, the $C_{0}$ complexity in Fig. 6(c) is obtained by varying parameter $h$ in the range $[0,1]$. In the $(a, h)$ plane, the chaos diagram based on the $C_{0}$ complexity of system (11) is calculated and shown in Fig. 7. It is worth noting that the complexity of system (11) increases with increasing parameters $a$ and $h$. From Fig. 7, it can be seen that the largest $C_{0}$ complexity of system (11) fluctuates within a small range for parameters $a$ and $h$. Based on the above discussion, I find that complexity analysis is a more convenient method to choose parameters for the discrete fractional map.

\section{Conclusions}

In this paper, I investigated the chaotic dynamics of the discrete reduced Lorenz map obtained by means of the discrete fractional calculus. As a result, a novel discrete reduced 
Lorenz map of fractional difference order is given in the form of an iteration formula. According to the phase diagrams and bifurcation diagrams, the paper has illustrated the presence of bifurcation and chaos in the discrete fractional reduced Lorenz map reported in literature. The discrete fractional reduced Lorenz map contains rich dynamical behaviors.

In addition, the $C_{0}$ complexity is calculated by utilizing the $C_{0}$ algorithm. These results show that the $C_{0}$ complexity algorithm is an efficient tool to choose parameters for practical applications of discrete fractional maps. Furthermore, an interesting phenomenon is that the $C_{0}$ complexity of discrete fractional system (11) increases with increasing fractional order $\alpha$.

It is hoped that the results reported here will be beneficial to researchers exploring other dynamical behaviors in the discrete fractional chaotic systems. The numerical evaluations are carried out with the software MATLAB.

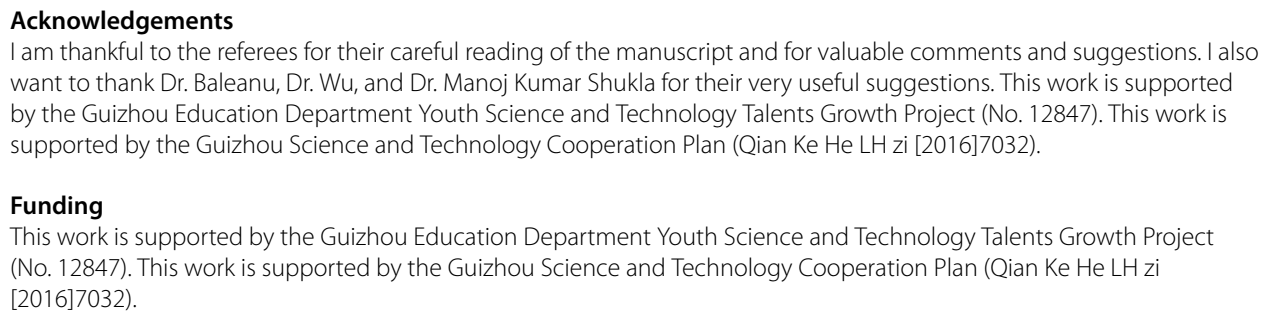

I am thankful to the referees for their careful reading of the manuscript and for valuable comments and suggestions. I also want to thank Dr. Baleanu, Dr. Wu, and Dr. Manoj Kumar Shukla for their very useful suggestions. This work is supported by the Guizhou Education Department Youth Science and Technology Talents Growth Project (No. 12847). This work is supported by the Guizhou Science and Technology Cooperation Plan (Qian Ke He LH zi [2016]7032).

\section{Funding}

This work is supported by the Guizhou Education Department Youth Science and Technology Talents Growth Project (No. 12847). This work is supported by the Guizhou Science and Technology Cooperation Plan (Qian Ke He LH zi [2016]7032).

\section{Availability of data and materials}

Not applicable.

\section{Competing interests}

The author declares that they have no competing interests.

\section{Author's contributions}

All authors contributed equally to the writing of this paper. All authors read and approved the final manuscript.

\section{Publisher's Note}

Springer Nature remains neutral with regard to jurisdictional claims in published maps and institutional affiliations.

Received: 21 March 2018 Accepted: 13 August 2018 Published online: 29 August 2018

\section{References}

1. Li, C.P., Peng, G.: Chaos in Chen's system with a fractional order. Chaos Solitons Fractals 22, $443-450$ (2004)

2. Li, C.P., Chen, G.: Chaos and hyperchaos in the fractional-order Rössler equations. Phys. A, Stat. Mech. Appl. 341, 55-61 (2004)

3. Grigorenko, I., Grigorenko, E.: Chaotic dynamics of the fractional Lorenz map. Phys. Rev. Lett. 91, 34101 (2003)

4. Atici, F.M., Eloe, P.W.: A transform method in discrete fractional calculus. Int. J. Difference Equ. 2, 165-176 (2007)

5. Xiao, H., Ma, Y., Li, C.: Chaotic vibration in fractional maps. J. Vib. Control 20, 964-972 (2014)

6. Atici, F.M., Eloe, P.W.: Initial value problems in discrete fractional calculus. Proc. Am. Math. Soc. 137, $981-989$ (2009)

7. Anastassiou, G.A.: About discrete fractional calculus with inequalities. In: Intelligent Mathematics: Computational Analysis. Springer, Berlin (2011)

8. Abdeljawad, T.: On Riemann and Caputo fractional differences. Comput. Math. Appl. 62, 1602-1611 (2011)

9. Chen, F., Luo, X., Zhou, Y.: Existence results for nonlinear fractional difference equation. Adv. Differ. Equ. 2011,713201 (2011)

10. Wu, G.C., Baleanu, D., Zeng, S.D.: Discrete chaos in fractional sine and standard maps. Phys. Lett. A 378, 484-487 (2014)

11. Wu, G.C., Baleanu, D.: Chaos synchronization of the discrete fractional logistic map. Signal Process. 102, 96-99 (2014)

12. Wu, G.C., Baleanu, D., Xie, H.P., Chen, F.L.: Chaos synchronization of fractional chaotic maps based on the stability condition. Phys. A, Stat. Mech. Appl. 460, 374-383 (2016)

13. Wu, G.C., Baleanu, D., Xie, H.P., Zeng, S.D.: Discrete fractional diffusion equation of chaotic order. Int. J. Bifurc. Chaos 26, 281-286 (2016)

14. Wu, G.C., Baleanu, D., Huang, L.L.: Novel Mittag-Leffler stability of linear fractional delay difference equations with impulse. Appl. Math. Lett. 82, 71-78 (2018)

15. Shukla, M.K., Sharma, B.B.: Investigation of chaos in fractional order generalized hyperchaotic Henon map. AEÜ, Int. J. Electron. Commun. 78, 265-273 (2017) 
16. Shukla, M.K., Sharma, B.B.: Stabilization of fractional order discrete chaotic systems. In: Fractional Order Control and Synchronization of Chaotic Systems, pp. 431-445 (2017)

17. Liu, Y:: Chaotic synchronization between linearly coupled discrete fractional Henon maps. Indian J. Phys. 90, 1-5 (2016)

18. Wu, G.C., Baleanu, D., Xie, H.P.: Riesz Riemann-Liouville difference on discrete domains. Chaos, Interdiscip. J. Nonlinear Sci. 26, $084308(2016)$

19. Wu, G.C., Baleanu, D.: Discrete chaos in fractional delayed logistic maps. Nonlinear Dyn. 80, 1697-1703 (2015)

20. Kumar, D., Singh, J., Baleanu, D.: A new analysis of the Fornberg-Whitham equation pertaining to a fractional derivative with Mittag-Leffler-type kernel. Eur. Phys. J. Plus 133, 70 (2018)

21. Kumar, D., Singh, J., Baleanu, D.: Analysis of regularized long wave equation associated with a new fractional operator with Mittag-Leffler-type kernel. Phys. A, Stat. Mech. Appl. 492, 155-167 (2018)

22. Kumar, D., Singh, J., Baleanu, D.: A new fractional model for convective straight fins with temperature-dependent thermal conductivity. Therm. Sci. 69, 96 (2017)

23. Singh, J., Kumar, D., Baleanu, D., Rathore, S.: An efficient numerical algorithm for the fractional Drinfeld-Sokolov-Wilson equation. Appl. Math. Comput. 335, 12-14 (2018)

24. Kumar, D., Singh, J., Baleanu, D.: A new numerical algorithm for fractional Fitzhugh-Nagumo equation arising in transmission of nerve impulses. Nonlinear Dyn. 91,1-11 (2018)

25. He, S., Sun, K., Wang, H.: Solution and dynamics analysis of a fractional order hyperchaotic system. Math. Methods Appl. Sci. 39, 2965-2973 (2016)

26. Shen, E.H., Cai, Z.J., Gu, F.J.: Mathematical foundation of a new complexity measure. Appl. Math. Mech. 26, 1188-1196 (2005)

27. Lorenz, E.N.: Computational chaos — a prelude to computational instability. Phys. D, Nonlinear Phenom. 35, 299-317 (1989)

28. Frouzakis, C.E., Kevrekidis, I.G., Peckham, B.B.: A route to computational chaos revisited: noninvertibility and the breakup of an invariant circle. Phys. D, Nonlinear Phenom. 177, 101-121 (2003)

29. Elabbasy, E.M., Elsadany, A.A., Zhang, Y.: Bifurcation analysis and chaos in a discrete reduced Lorenz map. Appl. Math. Comput. 228, 184-194 (2014)

\section{Submit your manuscript to a SpringerOpen ${ }^{\circ}$ journal and benefit from:}

- Convenient online submission

- Rigorous peer review

- Open access: articles freely available online

- High visibility within the field

- Retaining the copyright to your article

Submit your next manuscript at $\boldsymbol{\nabla}$ springeropen.com 